\title{
Comparison of free and bound volatile profiles of immature Litsea mollis fruits grown in five distinct regions of China
}

\author{
Miao LIANG ${ }^{1,2}$, Yan YANG ${ }^{1,2}$, Fu-Ping ZHENG ${ }^{3}$, Bao-Guo SUN ${ }^{3}$, Xing-Ping WANG ${ }^{1}$, Ai-Nong YU ${ }^{2,3, \star}$
}

\begin{abstract}
Immature Litsea mollis fruits (ILMFs) are widely used as cold appetizers and seasonings in China. The free and bound volatile components of ILMFs from five distinct regions of China were determined by gas chromatography-mass spectrometry (GC-MS) combined with headspace solid phase microextraction (SPME) in the present work. The results reveal sixty six free-form volatiles and fifty two bound-form volatiles in ILMFs and terpene hydrocarbons and oxygenated terpenes are the dominate components. In addition, sixty-seven volatile compounds are found in Litsea mollis fruit for the first time. The free $\alpha$-pinene, eucalyptol, linalool, $\alpha, \beta$-citral, geraniol and citronellal, and bound $\beta$-myrcene, $\alpha$-citral, geraniol, citronellal and eugenol are the major odor contributors of ILMFs. Multivariate statistical analysis indicates that the free and bound volatiles profiles of ILMFs of Fujian and Guangxi in southern China are similar and those of Yunnan and Sichuan in southwestern China are similar. The odor profiles of all of the ILMFs are floral, fruity and herbaceous.
\end{abstract}

Keywords: Litsea mollis; immature fruit; volatile; aroma; bound aroma compound.

Practical Application: The study provides valuable and reliable information of free and bound volatile aroma components in immature Litsea mollis fruits, and will help the development of natural foods for food industries.

\section{Introduction}

Litsea mollis Hemsl. is a member of the Lauraceae family. In China, it is mostly distributed in Guangdong, Guangxi, Fujian, Hunan, Hubei, Sichuan, Guizhou, Yunnan and eastern Tibet (Chinese Academy of Sciences, 2019, Figure S1). Litsea mollis Hemsl. is also found in some regions of Japan, Korea, New Zealand, North America, and South America (Huang et al., 2014). The large amounts of fatty acids and aromatic oils in its fruit make it of a great economic plant, especially for the production of drugs and lubricants (Kim et al., 2014). The volatile oil of ripe Litsea mollis fruits is a biopreservative of low-cost in the dairy industry (Cai et al., 2019).

Immature Litsea mollis fruit (ILMF) is generally pickled with soy sauce, vinegar, pepper, garlic, and ginger as a popular cold appetizer in southwestern China (Zhang, 2015). People usually only pay attention to the aroma of free volatiles in fruits, but the studies on the bound aroma compounds of other fruits show that the hydrolysis of bound volatiles can improve the overall aroma of the fruits. The bound forms, mainly referring to the glycosidically bound alcohols, terpenes, dimethyl isoprene, volatile organic acids and shikimic acid derivatives, can be released by enzymatic or acid hydrolysis (Sánchez-Palomo et al., 2017), and thus they are also considered as important aroma components (Sarry \& Gunata, 2004). The glycosides in the bound volatile compounds identified in fruits are mainly O- $\beta$-D-glucosides and O-diglycosides. O-glycoside hydrolases (EC 3.2.1.x) are a widespread group of enzymes of significant biological, biomedical and industrial importance. They, as well as fungal enzymes (usually from Aspergillus niger), can release the bound aroma (Davis \& Croteau, 2000).

Chen et al. (1984) extracted volatile oils from mature Litsea mollis fruits by steam distillation and found that the aroma of volatile oils mainly came from terpenoids. To the best of our knowledge, only one work has been reported on the free volatile compounds in ILMF (Yang et al., 2020), where six terpenoids are found to be responsible for the typical flavor of ILMF. Other than that, the aroma compounds of ILMF, either the free forms or bound forms, have been rarely studied.

In the present work, the volatile components in the ILMFs collected from different regions of China were extracted by headspace solid phase microextraction (SPME), and the glycosidically bound volatiles were enzymatically hydrolyzed with the pectinase from Aspergillus niger (Dziadas \& Jelen, 2016; Yang et al., 2019). SPME is a simple, low cost, solvent free and sensitive method for aroma compound extraction without heating required, and thus can minimize the loss of aroma. The volatile components were then identified and quantitatively analyzed by GC-MS, and the volatile profiles of the ILMFs were compared, aiming to provide a scientific foundation for understanding the

${ }^{2}$ School of Chemistry \& Environmental Engineering, Hubei Minzu University, Enshi, Hubei, China

${ }^{3}$ Beijing Advanced Innovation Center for Food Nutrition and Human Health, Beijing Technology and Business University (BTBU), Beijing, China

*Corresponding author: anyufc@163.com 
different free and bound aroma compositions of the ILMFs in different regions of China.

\section{Materials and methods}

\subsection{Materials and chemicals}

\section{ILMF samples}

Based on the main distribution areas of Litsea mollis published by the Chinese Academy of Sciences (Chinese Academy of Sciences, 2019), wild ILMFs with sizes of 3-4 mm were harvested in five main distribution areas in China including Fujian (FJ), Guangxi (GX), Hubei (HB), Yunnan (YN) and Sichuan (SC) provinces by a professional organization, Enshi Dongsheng Plant Development Co. Ltd. (Figure S1), and identified and validated by Professor Yong-Mei Yi at the School of Forestry and Horticulture, Hubei Minzu University. The samples were frozen immediately after collection, transported to the laboratory under frozen conditions in two days, and stored at $-78^{\circ} \mathrm{C}$ before processing and analysis. The detailed sampling protocol is provided in Table S1.

\section{Chemicals}

C7-C30 saturated alkanes reference standard, Aspergillus niger pectinase $\left(1.06 \mathrm{U} \mathrm{mg}^{-1}\right)$ and the standards of citral $(98.0 \%)$, a-pinene (98.0\%), a-phellandrene (85.0\%), (E)-2-hexen-1-ol (96.0\%), linalool (97.0\%), camphene (95.0\%), a-terpinene $(89.0 \%)$, allo-ocimene $(80.0 \%), \beta$-myrcene $(90.0 \%), \gamma$-terpinene $(97.0 \%)$, p-cymenene (98.0\%), citronellal (95.0\%), (Z)-verbenol (95\%), terpinolene $(85.0 \%)$, geraniol $(97.0 \%)$, nerol acetate $(98.0 \%)$, caryophyllene (98.0\%), 4-terpineol (95.0\%), a-terpineol (95.0\%), caryophyllene oxide (99.0\%), methyl cinnamate (99.0\%), methyl salicylate (99.0\%), L-perillaldehyde (92.0\%), nerolidol (98.0\%), carveol (95.0\%), humulene (96.0\%), nerol (97.0\%), eugenol (99.0\%), (-)-borneol (99.0\%), phenethanol (97.0\%), sulcatone (97.0\%), perilla alcohol (96.0\%), rose oxide $(99.0 \%)$, benzaldehyde (99.0\%), methyl o-anisate (99.0\%), eucalyptol (99.0\%), geranic acid $(85.0 \%)$ and L-pinocarveol (96.0\%) were obtained from Sigma-Aldrich Chemical Co. (St. Louis, MO, USA). All other chemicals were of analytical grade. Double-distilled water was used in all experiments.

\subsection{Juice preparation and free volatiles extraction}

The fruit samples were respectively thawed and juiced using a JYZ-E16 juicer (Joyoung Co., Ltd., Jinan, China). The juices were centrifuged at $4{ }^{\circ} \mathrm{C}$ at $8000 \mathrm{rpm}$ for $20 \mathrm{~min}$ in an Avanti J -30I centrifuge (Beckman Coulter Inc., Brea, USA). Ten milliliters of the supernatant of each juice were collected, mixed with $20.0 \mu \mathrm{L} 1$-octanol (1.5707g L $\mathrm{g}^{-1}$ in ethanol) internal standard and $2.0 \mathrm{~g} \mathrm{NaCl}$, transferred into a 20 -mL sample bottle containing a magnetic stirrer, sealed with a PTFE-silicon septum, and extracted by the method reported by Yang et al. (2020). Briefly, the sample was equilibrated at $46^{\circ} \mathrm{C}$ for $20 \mathrm{~min}$, and extracted with a DVB/CAR/PDMS (Divinylbenzene/Carboxen/ Polydimethylsiloxane) SPME fiber (50/30 $\mathrm{m}$; Supelco, Bellefonte, $\mathrm{PA}$ ) at $46^{\circ} \mathrm{C}$ for $36 \mathrm{~min}$. To ensure the accuracy of the analysis, three replicates of each juice sample were prepared and extracted.

\subsection{Isolation of bound volatile compounds}

The bound volatile compounds were extracted as described in our previous work (Yang et al., 2019). Briefly, $400 \mathrm{~mL}$ of juice supernatant was passed through a LC column $(400 \times 40 \mathrm{~mm})$ packed with $50 \mathrm{~g}$ Amberlite XAD-2 resin (20-60 mesh, Sigma Aldrich, St. Louis, Mo., USA) at the flow rate of $3 \mathrm{~mL} \mathrm{~min}{ }^{-1}$. The column was washed with double-distilled water and diethyl

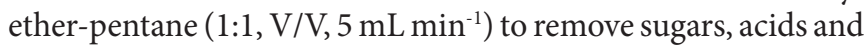
free aroma compounds. The bound compounds were then eluted with $600 \mathrm{~mL}$ methanol $(\geq 99.8 \%)$ at $5 \mathrm{~mL} \mathrm{~min}^{-1}$, dried under reduced pressure $(0.08 \mathrm{MPa})$ with a rotary evaporator (RE-52AA, Shanghai Yarong biochemical instrument factory, China) at $\leq 35^{\circ} \mathrm{C}$, and reconstituted in $40 \mathrm{~mL}$ of citric acid-disodium hydrogen phosphate buffer ( $\mathrm{pH} 5.5$, close to the $\mathrm{pH}$ of the juice). Free volatile residues were further removed by the liquid-liquid extraction with $80 \mathrm{~mL}$ of dichloromethane-pentane $(1: 1, \mathrm{~V} / \mathrm{V}) 3$ times. The aqueous phase was equally divided into four aliquots, and hydrolyzed with $150 \mathrm{mg}$ of Aspergillus niger pectinase $\left(1.06 \mathrm{U} \mathrm{mg}^{-1}\right)$ at $37^{\circ} \mathrm{C}$ for $48 \mathrm{~h}$. Each aliquot, two grams of $\mathrm{NaCl}$, a magnetic stirrer and $20 \mu \mathrm{L}$ of 1 -octanol (1.5707 $\mathrm{g} \mathrm{L}^{-1}$ in ethanol) were sealed in an extractor with PTFE-silicon septum and extracted by SPME as described in Section 2.2 for GC-MS analysis.

\subsection{Measurements of total soluble solids and pHs of ILMF juices}

The total soluble solids of ILMF juices were determined with a WYT-4 digital handheld refractometer (Beijing Yangtech Scientific Instruments Co., Ltd., Beijing, China) and their pHs were measured with a PB-21 pH meter (Sartorius AG Inc., Beijing, China) for the preparation of model juice. The results are listed in Supplementary material information Table S4.

\subsection{GC-MS analysis}

GC-MS analysis was conducted as described in our previous report (Yang et al., 2020) using a DB-5MS capillary column $(30 \mathrm{~m} \times 0.25 \mathrm{~mm}$ i.d $\times 0.25 \mu \mathrm{m}$, Agilent, Santa Clara, CA $)$ for GC separation.

\subsection{Quantification and odor activity values (OAVs) calculation}

To accurately quantify the volatile compounds, working standard solutions were prepared using a model juice containing $30.0 \mathrm{~g}$ sucrose and $30.0 \mathrm{~g}$ glucose per $1000 \mathrm{~mL}$ double-distilled water prepared based on the average total soluble solid and $\mathrm{pH}$ of ILMF juices as described in our previous report (Yang et al., 2019). The $\mathrm{pH}$ of the model juice was adjusted with citric acid solution to 5.34 (Table S4) for the preparation of free volatile standards solutions. The bound volatile standards solutions were prepared in citric acid-disodium hydrogen phosphate buffer ( $\mathrm{pH}$ 5.50). Each working standard was extracted with the same procedure as that for ILMF juice. The calibration curves with regression coefficients greater than 0.975 were established by SIM quantitative analysis (Table S2 \& Table S3). The volatile compounds with no standards available were quantified with the standard curves of the volatiles of similar functional groups and/or similar numbers of C. The results are 
reported as $\mu \mathrm{g} \mathrm{L}^{-1}$ juice. OAVs were calculated as described in our previous work (Yang et al., 2019).

\subsection{Statistical analyses}

All data are reported as mean value \pm standard deviation of three tests. Principle component analysis (PCA) and one-way ANOVA were conducted using the IBM SPSS Statistics 22 software (IBM, Armonk, NY, USA). Hierarchical clustering and heatmap visualization and plot were conducted with the TB tools (GitHub, 2021).

\section{Results and discussion}

\subsection{Free and bound volatile compounds identified in ILMFs}

Eighty two compounds including 66 free-forms and 52 bound-forms were identified in the ILMF samples by GC-MS (Table 1 and 2). Among them, 36 compounds are present in both free and bound forms. The number of compounds in ILMFs of the five regions displayed a high degree of similarity (Figure 1). Forty three free compounds (Figure 1A) and 34 bound compounds (Figure 1B) are found in the ILMFs of all five regions. Fourteen of them including $\alpha$-pinene, camphene, $\beta$-pinene, $\beta$-myrcene, p-cymenene, sulcatone, linalool, citronellal, 4-terpineol, borneol, $\alpha$-terpineol, $\beta$-citral, geraniol and $\alpha$-citral were also detected in the volatile oil of mature Litsea mollis fruit (Chen et al., 1984). Eucalyptol, linalool, $\alpha$-terpineol, $\beta$-citral, geraniol, and $\alpha$-citral were found in ILMFs by SPME in our previous work (Yang et al., 2020). Other 67 volatile compounds are detected in Litsea mollis fruit for the first time. These identified compounds are grouped as terpenoids, alcohols, esters and others for further discussion.

\section{Terpenoids}

Terpenoids including both terpene hydrocarbons and oxygenated terpenes are the major volatile compounds in ILMF, accounting for $84.8 \%$ and $78.8 \%$ of the total numbers of the free and bound volatiles, respectively. Terpenoids are secreted via the isoprenoid pathway in specific plant tissues (Wang et al., 2019a; Xiao et al., 2019). They are the most important aromatic compounds affecting the characteristics of fruits. There are abundant terpenoids in Lauraceae family (Niogret et al., 2013; Wang et al., 2019b). The nonvolatile precursors, especially the glycosidically bound ones, of terpenoids can be effectively hydrolyzed with enzyme to release the terpenoids (Swiegers \& Pretorius, 2005).

A total number of 28 free terpene hydrocarbons are detected, and the contents of $\alpha$-pinene, $\beta$-pinene, $\alpha$-terpinene, $\alpha$-terpinolene and $4-\mathrm{M}-3-(1-\mathrm{M})-\mathrm{C}$ are the highest (Table 1$)$. The total free terpene hydrocarbons content of the ILMF harvested in FJ is the highest with the value of $89488.51 \mu \mathrm{g} \mathrm{L} \mathrm{L}^{-1}$, followed by that of $\mathrm{HB}\left(80063.67 \mu \mathrm{g} \mathrm{L}^{-1}\right)$. The lowest is found in the ILMF of SC $\left(32036.66 \mu \mathrm{g} \mathrm{L}^{-1}\right)$. Camphene and $\alpha, \beta$-pinene, the terpenes widely found in plants, are the most important skeletons of naturally occurring bicyclic monoterpenes (Qiu et al., 2017). The ILMFs of GX contain the highest amount of sylvestrene with the value of $38045.93 \mu \mathrm{g} \mathrm{L}^{-1}$. Caryophyllene is a sesquiterpene hydrocarbon mainly found in pepper and some spices (Goas et al., 1978). Other free sesquiterpene hydrocarbons are rarely reported and their contributions to the aroma of Litsea mollis is negligible, which may explain their absence in the ripe fruit of Litsea mollis (Chen et al., 1984).

Fourteen bound terpene hydrocarbons are identified in the ILMFs (Table 2), and 11 of them are shared by all ILMFs of the five regions. $\beta$-Myrcene, sylvestrene, $\gamma$-terpinene and $\alpha$-terpinolene are the predominant bound terpene hydrocarbons. The bound terpene hydrocarbon content in the fruit of SC is the highest with the value of $22525.22 \mu \mathrm{g} \mathrm{L}{ }^{-1}$. Some sesquiterpenes are found in free form only, possibly because they are converted into other terpenoids during the hydrolysis. Further investigation on the formation and transformation mechanism of terpenoids in ILMFs is undergoing in our lab.

Oxygenated terpenes, mainly composed of alcohols, aldehydes and ketones, provide fruits many unique flavors (Stuart et al., 2001).
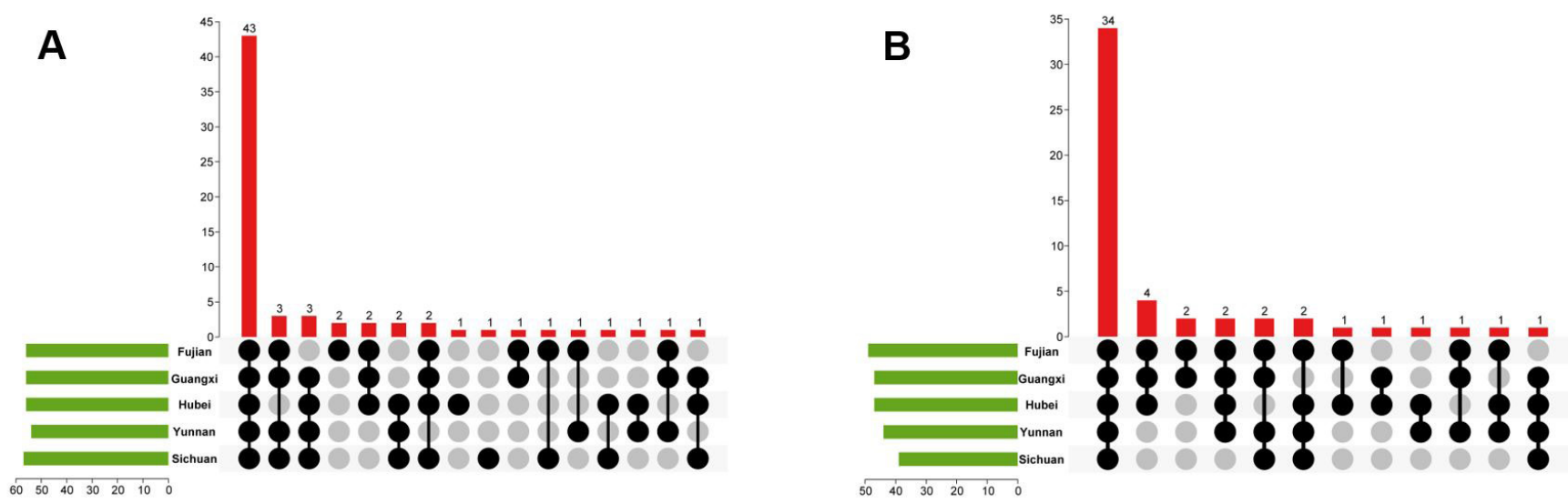

Figure 1. Common compounds in the ILMFs of five regions. (A) Upset plots of the free forms of odorants in the ILMFs of five regions; (B) Upset plot of the bound forms of odorants in the ILMFs of five regions. Green bars represent the number of volatile compounds identified in the ILMFs. Gray dots represent absence, while black dots represent the presence of the unique compounds. If more than one method contain the same unique compound, they are connected with lines. The number of the unique compounds presented individually or jointly is represented by red bars. 
Table 1. Free volatile compounds detected in the ILMFs of five distinct regions of China $\left(\mu g \mathrm{~L}^{-1}\right)$.

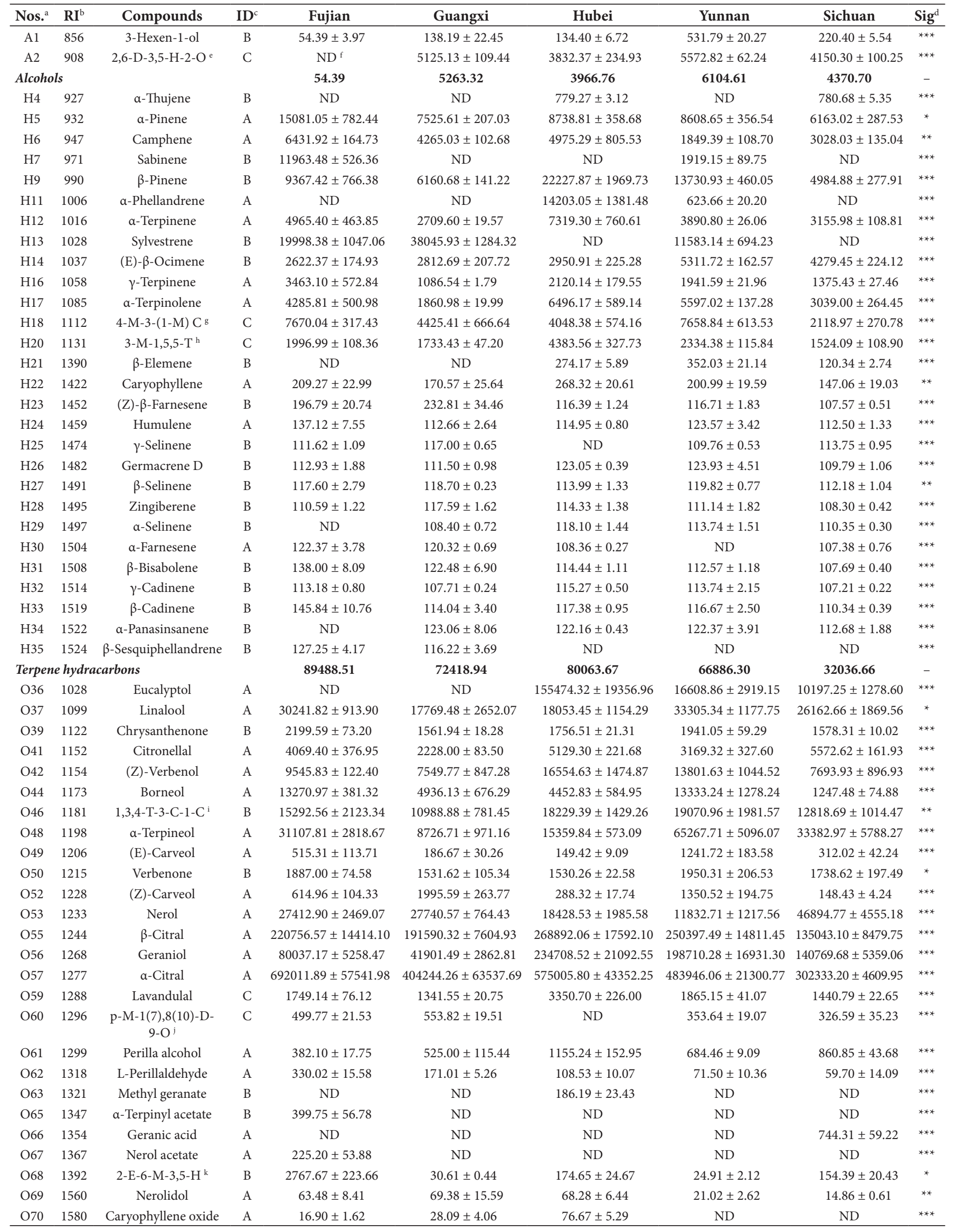


Table 1. Continued...

\begin{tabular}{|c|c|c|c|c|c|c|c|c|c|}
\hline Nos. $^{a}$ & $\mathbf{R I}^{\mathrm{b}}$ & Compounds & $\mathrm{ID}^{\mathrm{c}}$ & Fujian & Guangxi & Hubei & Yunnan & Sichuan & $\operatorname{Sig}^{\mathrm{d}}$ \\
\hline O71 & 1595 & Cedrol & B & $19.73 \pm 0.90$ & $19.79 \pm 2.72$ & $18.41 \pm 0.61$ & ND & ND & $* * *$ \\
\hline $\mathrm{O} 72$ & 1659 & Juniper camphor & B & $59.68 \pm 9.73$ & $58.42 \pm 9.10$ & $129.15 \pm 10.66$ & ND & $34.64 \pm 5.25$ & $* * *$ \\
\hline \multicolumn{4}{|c|}{ Oxygenated monoterpene } & 1135477.25 & 725749.10 & 1339280.99 & 1118947.88 & 729529.84 & - \\
\hline E74 & 1333 & Methyl o-anisate & A & $478.79 \pm 61.34$ & $104.75 \pm 3.67$ & $195.51 \pm 32.32$ & $341.58 \pm 65.59$ & $119.33 \pm 25.15$ & $* * *$ \\
\hline E75 & 1385 & Methyl cinnamate & A & $252.59 \pm 65.05$ & $331.98 \pm 17.95$ & ND & $2629.33 \pm 315.69$ & $90.89 \pm 14.62$ & $* * *$ \\
\hline Esters & & & & 7597.90 & 2437.11 & 3376.28 & 12919.72 & 3611.89 & - \\
\hline T77 & 886 & $2,6-\mathrm{D}-1,5-\mathrm{H}^{1}$ & B & $2308.02 \pm 196.65$ & $5194.48 \pm 1159.29$ & $5418.70 \pm 570.53$ & $5795.73 \pm 41.64$ & $1814.03 \pm 54.39$ & $* * *$ \\
\hline T79 & 985 & Sulcatone & A & ND & $1718.74 \pm 130.19$ & $14293.04 \pm 1038.42$ & ND & $1858.66 \pm 68.49$ & $* * *$ \\
\hline $\mathrm{T} 80$ & 1090 & p-Cymenene & A & $3625.01 \pm 484.14$ & $2364.51 \pm 338.63$ & $10991.11 \pm 613.30$ & $3209.60 \pm 498.54$ & $1668.70 \pm 296.17$ & $* * *$ \\
\hline $\mathrm{T} 82$ & 1446 & (E)-Isoeugenol & A & $114.97 \pm 20.21$ & ND & ND & ND & $52.71 \pm 3.31$ & $* * *$ \\
\hline Others & & & & 7371.71 & 10171.45 & 31632.56 & 9902.40 & 6301.21 & - \\
\hline
\end{tabular}

${ }^{\mathrm{a} A} \mathrm{~A}$-Alcohols, H-Terpenehydracarbons, O-Oxygenated terpenes, E-Esters, T-Others; ${ }^{\mathrm{R}}$ Retention indices; cReliability of the identification proposal: A, identified, mass spectrum, LRI agreed with literature data (National Institute of Standards and Technology, 2018) and LRI agreed with standards; ${ }^{\mathrm{B}}$, tentatively identified, mass spectrum agreed with the mass spectral database and LRI agreed with literature data (National Institute of Standards and Technology, 2018); ${ }^{\mathrm{C}}$, tentatively identified, mass spectrum agreed with the mass spectral database; ${ }^{\top}$ Sig: Statistical significance; ${ }^{*}$ Significant at $p<0.05$; ${ }^{*}$ Significant at $p<0.01$; ${ }^{* *}$ Significant at $p<0.001$, ns: no significant $(p>0.05)$; ${ }^{\mathrm{e}} 2,6-\mathrm{D}-3,5-\mathrm{H}-2-\mathrm{O}$ represents the compound of 2,6-dimethyl-3,5-heptadien-2-ol; ' ${ }^{\text {Not }}$ detected; ${ }^{8} 4-\mathrm{M}-3-(1-\mathrm{M}) \mathrm{C}$ represents the compound of 4-methyl-3-(1-methylethylidene)cyclohexene; ${ }^{\mathrm{b}} 3$-M-1,5,5-T represents the compound of 3-methylene-1,5,5-trimethylcyclohexene; ${ }^{\mathrm{i}} 1,3,4-\mathrm{T}-3$-C-1-C represents the compound of 1,3,4-trimethyl-3-cyclohexen-1-carboxaldehyde; ${ }^{j} \mathrm{p}-\mathrm{M}-1$ (7),8(10)-D-9-O represents

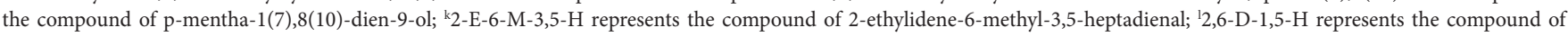
2,6-dimethyl-1,5-heptadiene.

Table 2. Bound volatile compounds found in the ILMFs of five distinct regions of China $\left(\mu \mathrm{g} \mathrm{L}^{-1}\right)$.

\begin{tabular}{|c|c|c|c|c|c|c|c|c|c|}
\hline Nos. $^{a}$ & $\mathbf{R I}^{\mathrm{b}}$ & Compounds & ID $^{c}$ & Fujian & Guangxi & Hubei & Yunnan & Sichuan & Sig $^{\mathrm{d}}$ \\
\hline A1 & 856 & 3-Hexen-1-ol & B & $23.22 \pm 1.41$ & $32.42 \pm 2.56$ & $51.92 \pm 1.98$ & $42.80 \pm 3.94$ & $\mathrm{ND}^{\mathrm{e}}$ & $* * *$ \\
\hline A3 & 1111 & Phenethanol & A & $3376.61 \pm 589.00$ & $1704.99 \pm 93.87$ & $2157.13 \pm 164.54$ & $2561.34 \pm 197.11$ & $2149.66 \pm 210.25$ & $* *$ \\
\hline \multicolumn{3}{|c|}{ Alcohols } & & 3399.82 & 1737.41 & 2209.05 & 2604.14 & 2149.66 & - \\
\hline H6 & 947 & Camphene & $\mathrm{A}$ & $50.47 \pm 7.65$ & $84.36 \pm 6.04$ & $11.42 \pm 1.29$ & $34.06 \pm 4.44$ & $99.01 \pm 5.16$ & $* * *$ \\
\hline $\mathrm{H} 8$ & 986 & a-Myrcene & B & $40.06 \pm 5.96$ & $44.74 \pm 3.26$ & $165.38 \pm 13.94$ & $167.48 \pm 20.84$ & $304.54 \pm 53.22$ & $* * *$ \\
\hline H10 & 991 & $\beta$-Myrcene & A & $2079.55 \pm 243.35$ & $2547.70 \pm 181.60$ & $3418.26 \pm 327.19$ & $3636.85 \pm 411.73$ & $5841.88 \pm 660.54$ & $* * *$ \\
\hline H11 & 1006 & a-Phellandrene & A & $336.82 \pm 30.72$ & $471.07 \pm 24.30$ & $445.74 \pm 22.25$ & $529.05 \pm 50.03$ & $664.70 \pm 63.59$ & $* * *$ \\
\hline H12 & 1016 & $\alpha$-Terpinene & A & $633.19 \pm 33.98$ & $845.26 \pm 58.20$ & $993.24 \pm 103.08$ & $1076.81 \pm 125.09$ & $1549.18 \pm 176.84$ & $* * *$ \\
\hline H13 & 1028 & Sylvestrene & B & $796.38 \pm 99.09$ & $2087.43 \pm 87.46$ & $2054.42 \pm 154.27$ & $4532.16 \pm 484.39$ & $5733.13 \pm 558.04$ & $* * *$ \\
\hline H14 & 1037 & (E)- $\beta$-Ocimene & B & $414.42 \pm 45.45$ & $841.87 \pm 42.79$ & $536.46 \pm 52.68$ & $1272.24 \pm 153.30$ & $1610.46 \pm 167.60$ & $* * *$ \\
\hline H15 & 1047 & (Z)- $\beta$-Ocimene & B & $690.31 \pm 74.63$ & $1375.89 \pm 121.03$ & $1130.98 \pm 65.37$ & $1305.19 \pm 153.69$ & $3344.49 \pm 360.19$ & $* * *$ \\
\hline H16 & 1058 & $\gamma$-Terpinene & A & $1376.87 \pm 103.89$ & $1022.65 \pm 39.48$ & $1732.48 \pm 279.44$ & $1096.33 \pm 125.50$ & $994.57 \pm 107.61$ & $* *$ \\
\hline H17 & 1085 & $\alpha$-Terpinolene & A & $714.10 \pm 69.94$ & $1295.32 \pm 66.48$ & $1058.56 \pm 88.80$ & $1676.15 \pm 186.17$ & $2033.15 \pm 190.86$ & $* * *$ \\
\hline H19 & 1128 & allo-Ocimene & A & $155.29 \pm 28.65$ & $131.29 \pm 12.44$ & $215.21 \pm 19.43$ & $215.90 \pm 28.41$ & $338.24 \pm 45.09$ & $* * *$ \\
\hline $\mathrm{H} 22$ & 1422 & Caryophyllene & A & $8.07 \pm 0.93$ & ND & $14.94 \pm 1.46$ & $23.96 \pm 6.62$ & $11.85 \pm 2.04$ & $* * *$ \\
\hline $\mathrm{H} 23$ & 1452 & $(Z)$ - $\beta$-Farnesene & B & $36.16 \pm 6.04$ & $17.11 \pm 3.28$ & $3.29 \pm 0.53$ & ND & ND & $* * *$ \\
\hline $\mathrm{H} 24$ & 1459 & Humulene & A & ND & ND & $15.45 \pm 1.62$ & $29.48 \pm 7.59$ & ND & $* * *$ \\
\hline \multicolumn{3}{|c|}{ Terpene hydracarbons } & & 7331.71 & 10764.69 & 11795.83 & 15595.67 & 22525.22 & - \\
\hline $\mathrm{O} 37$ & 1099 & Linalool & A & $85.04 \pm 5.02$ & $78.46 \pm 2.36$ & $112.68 \pm 6.44$ & $75.98 \pm 6.02$ & $129.11 \pm 9.73$ & $* * *$ \\
\hline O38 & 1120 & (Z)-p-2,8-Menthadien-1-ol & B & $9.44 \pm 0.71$ & $15.06 \pm 0.20$ & $8.03 \pm 0.29$ & $11.18 \pm 0.88$ & $14.86 \pm 0.70$ & $* * *$ \\
\hline $\mathrm{O} 40$ & 1151 & Nerol oxide & B & $121.35 \pm 6.08$ & $117.18 \pm 9.33$ & $745.36 \pm 91.09$ & ND & ND & $* * *$ \\
\hline $\mathrm{O} 41$ & 1152 & Citronellal & A & $229.83 \pm 17.08$ & $218.12 \pm 26.20$ & $1982.50 \pm 352.45$ & $314.36 \pm 41.53$ & $313.86 \pm 29.90$ & $* * *$ \\
\hline O42 & 1154 & (Z)-Verbenol & A & $147.57 \pm 24.41$ & $15.60 \pm 2.02$ & ND & $80.63 \pm 11.74$ & $38.57 \pm 3.93$ & $* * *$ \\
\hline $\mathrm{O} 43$ & 1162 & Pinocarvone & B & $320.65 \pm 62.67$ & $38.42 \pm 0.85$ & $75.32 \pm 8.62$ & ND & ND & $* * *$ \\
\hline $\mathrm{O} 44$ & 1173 & Borneol & A & $2304.02 \pm 197.03$ & $2354.00 \pm 278.98$ & $1932.86 \pm 217.31$ & $1607.55 \pm 146.89$ & $1833.93 \pm 110.94$ & * \\
\hline $\mathrm{O} 45$ & 1180 & 4-Terpineol & A & $613.49 \pm 75.32$ & $328.35 \pm 13.93$ & $292.40 \pm 23.01$ & $254.10 \pm 8.93$ & $246.44 \pm 4.90$ & $* * *$ \\
\hline $\mathrm{O} 47$ & 1186 & 3,9-Epoxy-1-p-menthene & B & $109.00 \pm 6.13$ & $57.08 \pm 2.35$ & $43.55 \pm 0.21$ & $68.77 \pm 2.09$ & ND & $* * *$ \\
\hline $\mathrm{O} 48$ & 1198 & a-Terpineol & $\mathrm{A}$ & $490.72 \pm 43.54$ & $513.80 \pm 59.08$ & $749.72 \pm 102.77$ & $296.59 \pm 28.35$ & $474.35 \pm 36.65$ & $* * *$ \\
\hline O49 & 1206 & (E)-Carveol & A & $82.43 \pm 11.88$ & $170.31 \pm 8.66$ & ND & ND & ND & $* * *$ \\
\hline $\mathrm{O} 51$ & 1219 & p-Menth-1-en-9-al & B & $1185.42 \pm 77.94$ & $498.02 \pm 46.03$ & $1924.39 \pm 262.25$ & $288.27 \pm 18.06$ & $339.91 \pm 59.71$ & $* * *$ \\
\hline O52 & 1228 & (Z)-Carveol & A & $46.04 \pm 0.94$ & $44.11 \pm 6.03$ & ND & ND & ND & $* * *$ \\
\hline $\mathrm{O} 53$ & 1233 & Nerol & A & $1188.39 \pm 52.83$ & $612.35 \pm 5.57$ & $825.21 \pm 99.94$ & $842.40 \pm 69.77$ & $3737.01 \pm 231.89$ & $* * *$ \\
\hline O54 & 1237 & 2-Hydroxycineol & B & $43.72 \pm 5.40$ & ND & $21.30 \pm 0.78$ & $47.32 \pm 1.92$ & ND & $* * *$ \\
\hline O55 & 1244 & $\beta$-Citral & A & $1436.22 \pm 159.82$ & $144.82 \pm 2.55$ & $193.62 \pm 14.69$ & $875.16 \pm 1140.35$ & $1436.57 \pm 158.83$ & $* * *$ \\
\hline O56 & 1268 & Geraniol & A & $2886.74 \pm 95.24$ & $3962.00 \pm 543.32$ & $5033.78 \pm 43.12$ & $5244.25 \pm 761.23$ & $19398.16 \pm 2427.27$ & $* * *$ \\
\hline
\end{tabular}


Table 2. Continued...

\begin{tabular}{|c|c|c|c|c|c|c|c|c|c|}
\hline Nos. $^{\mathrm{a}}$ & $\mathbf{R I}^{\mathrm{b}}$ & Compounds & ID $^{\mathrm{c}}$ & Fujian & Guangxi & Hubei & Yunnan & Sichuan & Sig $^{\mathrm{d}}$ \\
\hline O57 & 1277 & $\alpha$-Citral & A & $813.31 \pm 106.83$ & $1200.86 \pm 104.72$ & $358.29 \pm 20.75$ & $5187.31 \pm 651.11$ & $4059.58 \pm 478.43$ & $* * *$ \\
\hline O58 & 1279 & Phellandral & B & $48.70 \pm 1.19$ & ND & $70.96 \pm 15.19$ & $37.68 \pm 4.16$ & $191.86 \pm 13.52$ & $* * *$ \\
\hline $\mathrm{O} 60$ & 1296 & p-M-1(7),8(10)-D-9-O ${ }^{f}$ & $\mathrm{C}$ & $591.06 \pm 54.50$ & $538.13 \pm 48.36$ & $186.98 \pm 21.89$ & $393.98 \pm 36.80$ & $387.02 \pm 47.48$ & $* * *$ \\
\hline O61 & 1299 & Perilla alcohol & A & $58.12 \pm 3.22$ & $26.55 \pm 3.87$ & $33.72 \pm 2.45$ & $33.18 \pm 1.76$ & $47.80 \pm 3.45$ & $* * *$ \\
\hline $\mathrm{O} 63$ & 1321 & Methyl geranate & B & $1253.60 \pm 183.20$ & $939.75 \pm 115.87$ & $473.06 \pm 4.73$ & $428.06 \pm 51.85$ & $393.47 \pm 80.23$ & $* *$ \\
\hline O64 & 1331 & p-Mentha-1,4-dien-7-ol & B & $182.23 \pm 15.00$ & $23.95 \pm 0.88$ & $29.72 \pm 5.16$ & $22.59 \pm 0.81$ & $34.92 \pm 2.40$ & $* * *$ \\
\hline $\mathrm{O} 65$ & 1347 & $\alpha$-Terpinyl acetate & B & $136.12 \pm 15.77$ & $35.90 \pm 3.47$ & $41.57 \pm 6.31$ & ND & ND & $* * *$ \\
\hline O66 & 1354 & Geranic acid & A & $902.51 \pm 110.65$ & $46.94 \pm 0.81$ & $30.49 \pm 1.70$ & $36.24 \pm 1.53$ & $28.44 \pm 0.57$ & $* * *$ \\
\hline O67 & 1367 & Nerol acetate & A & $112.33 \pm 10.62$ & ND & $34.16 \pm 1.70$ & ND & ND & $* * *$ \\
\hline O68 & 1392 & 2-E-6-M-3,5-H ${ }^{g}$ & B & $62.58 \pm 4.48$ & $10.24 \pm 0.49$ & $5.92 \pm 0.21$ & $29.57 \pm 2.38$ & $28.87 \pm 2.08$ & $* * *$ \\
\hline \multicolumn{4}{|c|}{ Oxygenated terpenes } & 15460.63 & 11990.00 & 15205.59 & 16175.17 & 33134.74 & - \\
\hline E73 & 1192 & Methyl salicylate & A & $2027.92 \pm 408.25$ & $2758.99 \pm 145.34$ & $1212.37 \pm 307.04$ & $2411.68 \pm 302.71$ & $2253.22 \pm 91.40$ & $* *$ \\
\hline E74 & 1333 & Methyl o-anisate & A & $171.10 \pm 6.76$ & $39.73 \pm 4.42$ & $24.72 \pm 3.28$ & $25.59 \pm 2.14$ & $23.97 \pm 2.27$ & $* * *$ \\
\hline E75 & 1385 & Methyl cinnamate & A & $2125.60 \pm 243.98$ & $110.18 \pm 1.38$ & $184.89 \pm 28.60$ & $982.44 \pm 155.18$ & $410.07 \pm 77.46$ & $* * *$ \\
\hline Esters & & & & 4324.62 & 2908.90 & 1421.98 & 3419.71 & 2687.27 & - \\
\hline T77 & 886 & $2,6-\mathrm{D}-1,5-\mathrm{H}^{\mathrm{h}}$ & B & $127.83 \pm 9.17$ & $68.90 \pm 1.73$ & ND & $127.01 \pm 6.92$ & $65.66 \pm 0.29$ & $* * *$ \\
\hline T78 & 964 & Benzaldehyde & A & $60.71 \pm 12.45$ & $4.28 \pm 0.75$ & ND & $15.45 \pm 1.65$ & ND & *** \\
\hline T79 & 985 & Sulcatone & A & ND & $0.39 \pm 0.00$ & $0.39 \pm 0.00$ & ND & ND & ** \\
\hline $\mathrm{T} 80$ & 1090 & p-Cymenene & A & $260.75 \pm 44.39$ & $244.72 \pm 17.56$ & $181.15 \pm 17.35$ & $258.48 \pm 37.74$ & $264.56 \pm 20.41$ & ns \\
\hline $\mathrm{T} 81$ & 1352 & Eugenol & A & ND & $105.26 \pm 15.12$ & $333.52 \pm 19.12$ & $998.14 \pm 119.42$ & $939.70 \pm 219.04$ & $* * *$ \\
\hline $\mathrm{T} 82$ & 1446 & (E)-Isoeugenol & A & $73.58 \pm 7.71$ & $129.07 \pm 23.45$ & $153.01 \pm 19.91$ & $248.57 \pm 27.11$ & $386.90 \pm 63.99$ & $* * *$ \\
\hline Others & & & & 522.87 & 552.62 & 668.06 & 1647.65 & 1656.82 & - \\
\hline
\end{tabular}

${ }^{\mathrm{a} A} \mathrm{~A}$-Alcohols, H-Terpenehydracarbons, O-Oxygenated terpenes, E-Esters, T-Others; ${ }^{\mathrm{b}}$ Retention indices; cReliability of the identification proposal: A, identified, mass spectrum, LRI agreed with literature data (National Institute of Standards and Technology, 2018) and LRI agreed with standards; ${ }^{\circledR}$, tentatively identified, mass spectrum agreed with the mass spectral database and LRI agreed with literature data (National Institute of Standards and Technology, 2018); ${ }^{\mathrm{C}}$, tentatively identified, mass spectrum agreed with the mass spectral database; ${ }^{\mathrm{d} S}$ Sig: Statistical significance; ${ }^{*}$ Significant at $p<0.05$; ${ }^{*}$ Significant at $p<0.01$; ${ }^{* *}$ Significant at $p<0.001$, ns: no significant $(p>0.05)$; ${ }^{\mathrm{C}}$ Not detected; ${ }^{\mathrm{f}} \mathrm{p}-\mathrm{M}-1(\mathrm{l}), 8(10)$-D-9-O represents

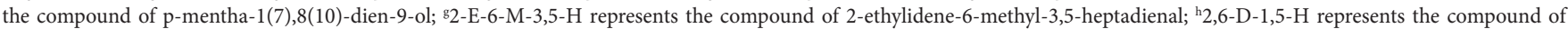
2,6-dimethyl-1,5-heptadiene.

In addition, they can show better antimicrobial activities than hydrocarbons (Guimarães et al., 2019). A total number of 37 free and bound oxygenated terpenes are found in the ILMFs, which include 28 free forms dominated by linalool, 1,3,4-T-3-C-1-C, $\alpha$-terpineol, nerol, $\beta$-citral, geraniol and $\alpha$-citral. Linalool is an important fruity and floral terpenoid that is usually produced by hydrolysis, redox reactions, and rearrangement (Davis \& Croteau, 2000; Qian \& Wang, 2005). Citral is the most abundant compound among these free oxygenated terpenes, accordant with the results reported in our previous study (Yang et al., 2020). The total content of $\alpha$-citral and $\beta$-citral accounts for $60-82 \%$ of the total free oxygenated terpenes for all the fruits from different regions. Citral possesses excellent antibacterial activities, and has been used in preservatives, soap and cosmetics industries (Onawunmi, 2008). It is worth noting that free eucalyptol is only detected in the fruits of $\mathrm{HB}, \mathrm{YN}$ and SC, with the highest content of $155474.32 \mu \mathrm{g} \mathrm{L}^{-1}$ in the ILMF of HB. Its absence in the ILMFs of FJ and GX may be explained with their geographic locations. HB, YN and SC are in the southwestern China, and FJ and GX are located in the southern China.

All bound oxygenated terpenes, except for borneol and geraniol, are present in the ILMFs at low levels. The highest total content of bound oxygenated terpenes is found in the ILMF of $\mathrm{SC}$ with the value of $33134.74 \mu \mathrm{g} \mathrm{L}-1$, which is mainly attributed to the high contents of nerol, geraniol and $a$-citral. Releasing the flavor compounds is generally positive for the overall aroma, as most odoriferous terpene alcohols, such as geraniol, nerol, citronellol and linalool, can enhance floral aroma (Dziadas \& Jelen, 2016).

Eighteen oxygenated terpenes including linalool, citronellal, (Z)-verbenol and borneol are found in both free and bound forms. $a$-Terpineol is a monoterpene alcohol with fruity aroma, and has been extensively reported in fruits and vegetables (Sharma et al., 2010). Bornol is a reduction product of camphor, which emits a peppery and peppermint odor. Geraniol can contribute notably to the aroma of ILMF, yet only trace amount found in the volatile oil of mature Litsea mollis fruit (Chen et al., 1984). It has also been found in lychee (Chyau et al., 2003), kiwifruit (Garcia et al., 2011), black velvet tamarind (Lasekan \& See, 2015) and other fruits.

\section{Alcohols and esters}

Three alcohols including 3-hexen-1-ol, 2,6-D-3,5-H-2-O and phenethanol are identified in the ILMFs. 3-Hexen-1-ol is an important C6 compound. It has also been found in the Spanish cultivars of pomegranate (Melgarejo et al., 2011). It is both in free and bound forms in all ILMFs, except for those of SC in the bound form. 2,6-D-3,5-H-2-O is present in all ILMFs only in the free form at very high contents ranging from 3832.37-5572.82 $\mu \mathrm{g} \mathrm{L}^{-1}$.

Esters are the major contributors of the fruity and sweet odor notes of fruits and vegetables (Wu et al., 2016). They can be produced by the enzymatic reactions of alcohols and acyl $\mathrm{CoA}^{\prime} \mathrm{s}$ 
derived from the fatty acid and amino acid metabolism (Wyllie \& Fellman, 2000). Three esters including methyl salicylate, methyl $\mathrm{o}$-anisate and methyl cinnamate are detected in the ILMFs, which are not reported in the volatile oil of mature Litsea mollis fruit (Chen et al., 1984). The content of free ester methyl salicylate varies with the geographic origin of the fruit, with the highest value of $9948.81 \mathrm{~g} \mathrm{~L}^{-1}(\mathrm{YN})$. The bound forms of the esters are also found in all the ILMFs with the highest total content in the ILMFs of FJ (4324.62 $\left.\mu \mathrm{g} \mathrm{L}^{-1}\right)$ and the lowest in the ILMFs harvested in $\mathrm{HB}\left(1421.98 \mu \mathrm{g} \mathrm{L}^{-1}\right)$.

\section{Others}

Seven compounds including three norisoprenoids, two phenols, one aldehyde and one aromatic hydrocarbon are classified as other compounds. They are detected in Litsea mollis fruit for first time (Chen et al., 1984). The content of 2,6-D-1,5-H ranges from $1814.03 \mu \mathrm{g} \mathrm{L} \mathrm{L}^{-1}$ to $5795.73 \mu \mathrm{g} \mathrm{\textrm {L } ^ { - 1 }}$ for the free form and the highest content is only $127.83 \mu \mathrm{g} \mathrm{L}^{-1}$ for the bound form. Sulcatone is an oxidation byproduct or degradation product of lycopene, $a$-farnesene, citral, or conjugated trienols (Liu et al., 2018). It has also been detected in apple (Whitaker \& Saftner, 2000). High contents of 2,5-dihydrotoluene are present in all ILMFs, but in the free form only. High contents of bound eugenol are found in the ILMFs of YN $\left(998.14 \mu \mathrm{g} \mathrm{L}^{-1}\right)$ and SC $\left(939.70 \mu \mathrm{g} \mathrm{L}^{-1}\right)$. The contents of free form of (E)-isoeugenol are low, but high amounts of bound form are found in the ILMFs of SC, suggesting that glucoside bound eugenol and isoeugenol can be hydrolyzed into their free forms with pectinase, and the natural ILMF lacks hydrolysis enzymes of the bound eugenol and isoeugenol. Benzaldehyde is in bound forms in the ILMFs. However, these volatiles account for a very small proportion of the total volatile.

\subsection{Odor profiles of ILMFs of different regions}

$\mathrm{OAV}$ is an important parameter evaluating the contribution of a volatile compound to food odor. Table 3 lists the OAVs, odor descriptions and aromatic series of the volatile compounds found in the ILMFs. The overall odors are classified into six categories including fruity, floral, sweet, herbaceous, woody and chemical. Thirty-three volatiles found in the ILMFs exhibit OAVs greater than 1, and thus are considered as the odor contributors. The bound aroma compounds can contribute to the odor of ILMFs only after they are released by enzymes or acids, thus indirectly.

Terpene hydrocarbons and oxygenated terpenes, such as $\alpha$-pinene, eucalyptol, linalool, $\alpha, \beta$-citral, geraniol and citronellal, are the major free volatiles contributing to the odor of ILMF. The contributions of the alcohols are negligible due to the high odor threshold of 3-hexen-1-ol. $\alpha, \beta$-Citral and citronellal enhance the fruity aroma, and the floral scent is mainly attributed to geraniol. Linalool, commonly found in citrus fruit, flowers and spice plants, is responsible for the pleasant fruity and floral odor notes of ILMF due to its low odor threshold in water (Buettner \& Schieberle, 2001; Elmaci \& Altug, 2005). The herbaceous odor mainly originates from eucalyptol and a-pinene, especially in the ILMFs of HB where eucalyptol displays the highest OAV of
2429.29. The odors completely opposite to the odor of ILMF, such as muddy and oily odors, are grouped as "chemical" odor, which also significantly affect fruit odor. Although there are abundant $\gamma$-terpinene and $\alpha$-terpineol in all ILMFs samples, they make no significant contributes to the fruit odor because of their high odor thresholds. The bound aroma compounds that contribute to the odor of ILMF mainly include $\beta$-myrcene (OAV, 57.77-162.27), a-citral (OAV, 11.20-126.86), geraniol (OAV, 72.17-484.95), citronellal (OAV, 36.35-330.42) and eugenol (OAV, 17.54-166.36).

The odor vectors of the free and bound aroma compounds are plotted based on their aromatic series and the sum of their OAVs in natural logarithmic scale (Figure 2). It is found that the overall odor profiles of the free aroma compounds detected in the ILMFs of the five regions are similar, predominated by floral and fruity odors that are usually derived from the free volatiles rather than the bound volatiles, followed by herbaceous odor (Figure 2A). The effects of sweet and woody odors on the ILMF aroma are also similar. Most aroma characteristics of the ILMFs of $\mathrm{HB}$ are more pronounced than those of other regions. The bound volatiles also exhibit floral, fruity and herbaceous odor profiles, but with stronger sweet and woody odors than those of free forms (Figure 2B). It explains that the sweet and woody odor of ILMF juice becomes stronger after the enzymatic hydrolysis. All odors of the bound volatiles, except for the fruity odor, of the ILMFs collected in SC are notably stronger than those of other regions, which is closely related to the highest altitude, less sunshine and low temperature in SC (Table S1). The herbaceous, sweet, woody and floral odors are more evident in the ILMFs of YN and SC. It is worth noting that the OAVs of the bound aroma compounds with chemical odor is very low or even negligible, and thus enzyme or acid hydrolysis does not increase chemical odor.

\subsection{Multivariate statistical analysis of free and bound volatile compounds}

PCA

To better understand the similarity of the ILMFs from five distinct regions of China, PCA analysis was conducted using 66 free and 52 bound volatile compounds found in the ILMFs as the variables for dimensionality reduction without losing much information (Shin et al., 2010. Figure 3).

Figure 3A shows the first two principal components ( $\mathrm{PC} 1$ and PC2) of the free volatile compounds respectively with $63.82 \%$ and $35.18 \%$ of data variances. The score distributions in PC1 and PC2 divide the ILMFs of the five regions into two groups. The five regions are all positioned at the right side of the loading plot, indicating that their high scores in PC1. FJ and GX are positively correlated to PC2, suggesting that the similarity of the ILMFs in these two regions is high. The free volatile compounds of the samples harvested in SC, $\mathrm{YN}$ and $\mathrm{HB}$ are close to each other and are negatively correlated to PC2, suggesting that their free volatile profiles are similar.

The bound volatile compounds are also positively correlated to PC1 (Figure 3B), and those of YN and SC are still on the PC2 negative axis. However, unlike the free volatile compounds, the 


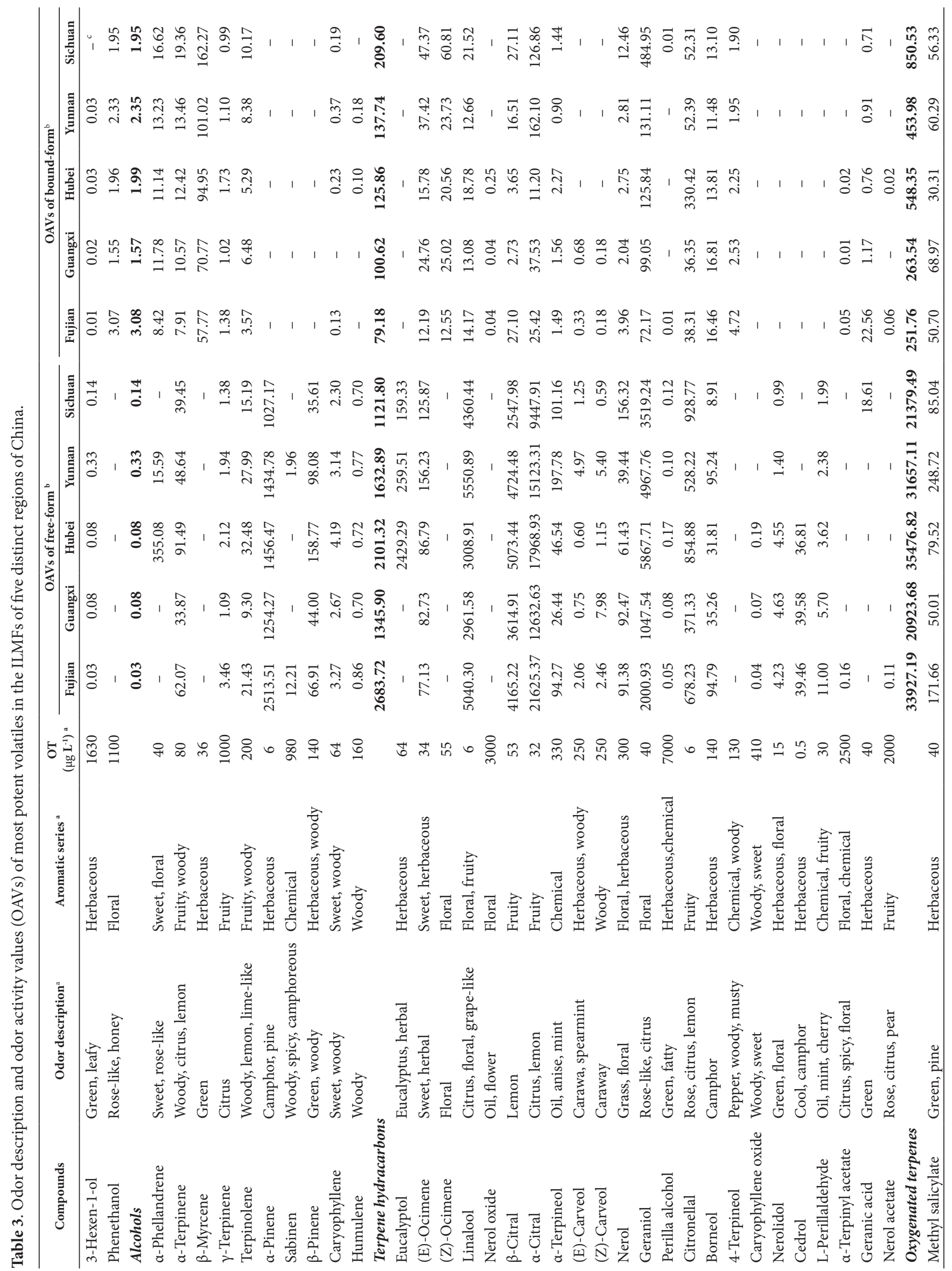




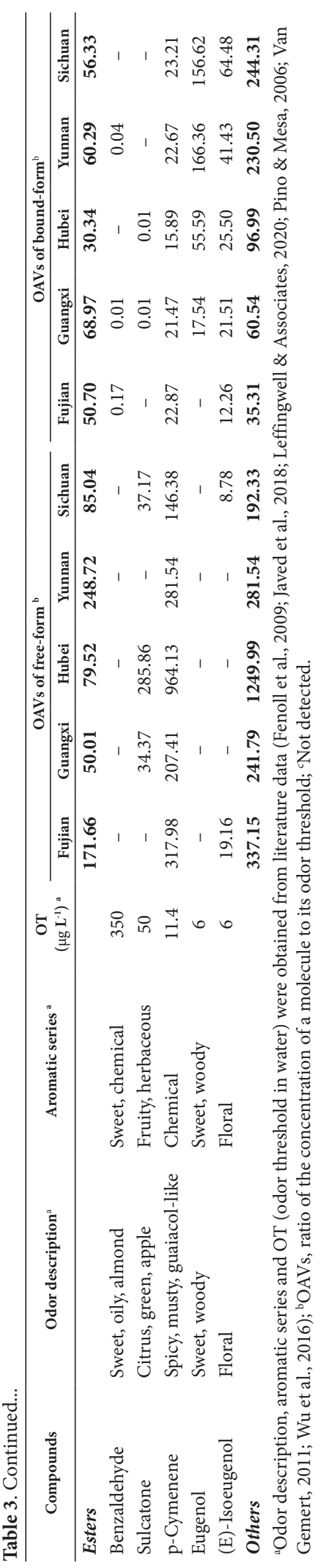


hydrolyzed bound volatiles in the ILMFs of HB are positively correlated with PC2, similar to the bound volatile profile of the ILMFs in GX. The bound volatile compounds found in the ILMF of FJ exhibit high scores in positive PC2. Phenethanol (A3), (Z)-verbenol (O42), p-M-1(7),8(10)-D-9-O (O60), p-mentha1,4-dien-7-ol (O64), geranic acid (O66), methyl o-anisate (E74), methyl cinnamate (E75) and benzaldehyde (T78) are positively related to FJ, indicating that these compounds are abundant in the ILMFs of FJ. Unlike the free volatile profile, the bound aroma profile is mainly correlated to oxygenated terpenes (Figure 3B).

\section{Cluster analysis}

To further distinguish the free and bound volatile compounds of the ILMFs from different geological regions, all the identified volatile compounds were subjected to hierarchical clustering.
A

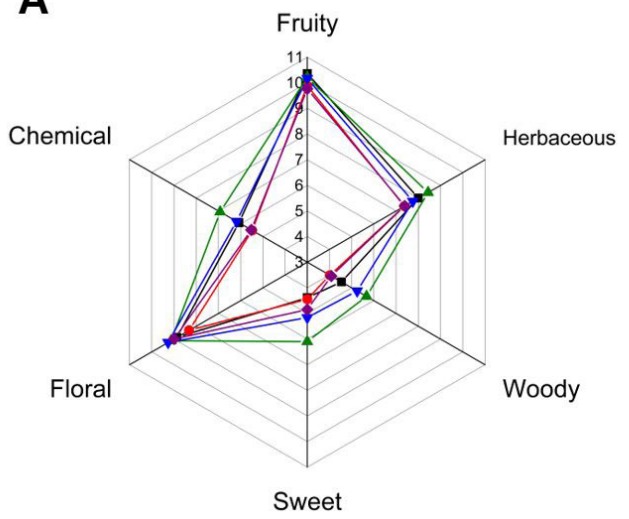

B

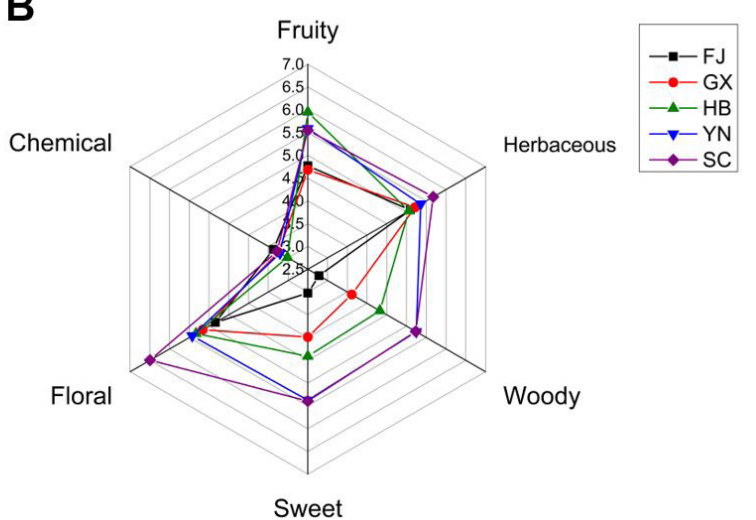

Figure 2. Aromatic plots of free (A) and bound (B) aroma compounds according to its odor activity values (in natural logarithmic scale). The colors of lines represent different odor profiles of the ILMFs in the five regions.
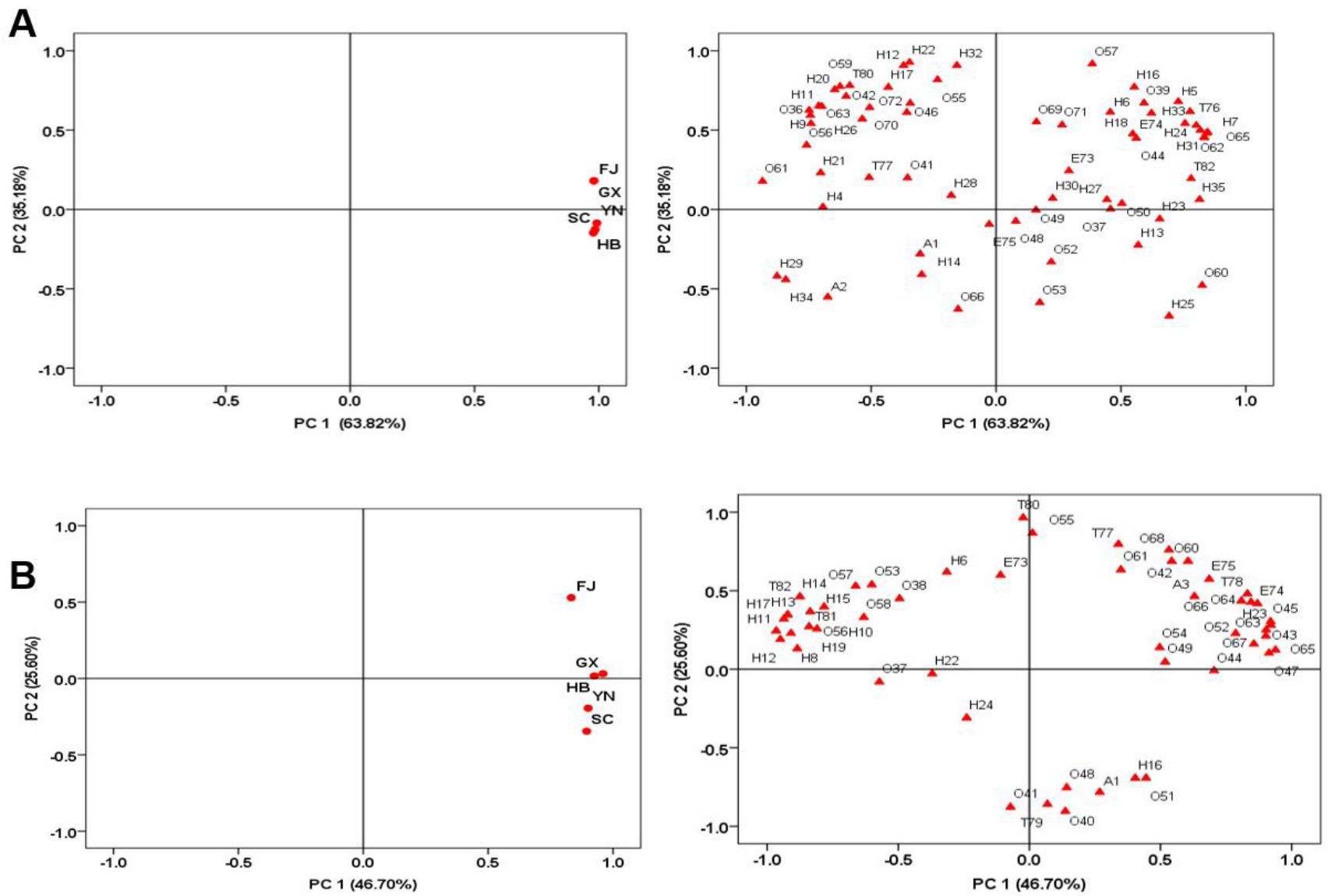

Figure 3. PCA of free (A) and bound (B) volatile compounds found in ILMFs. Compound nos. correspond to Table 1 and Table 2. 
The clustering of the free volatile compounds divides the ILMFs of the five regions into two groups (Figure 4). GX and FJ are in the same group for their similar contents of free volatiles. $\alpha$-Thujene, $\beta$-pinene, $\alpha$-phellandrene, $\beta$-elemene, eucalyptol, methyl geranate and geranic acid are absent in the ILMFs of these two regions, and their contents of (Z)- $\beta$-farnesene, $\beta$-citral and juniper camphor are similar. The ILMFs of SC are assembled with those of $\mathrm{HB}$ and $\mathrm{YN}$ because of the high resemblance of their free volatile profiles, especially their similar eucalyptol concentrations.

The ILMFs of the different regions are also divided into two groups by the clustering of their bound volatile compositions, with $\mathrm{FJ}$ and $\mathrm{GX}$ as one group for their similar contents of $\alpha$-myrcene, $\beta$-myrcene, allo-ocimene, $\alpha$-citral and p-M-1(7),8(10)-D-9-O. The other group of $\mathrm{HB}, \mathrm{GX}$ and FJ shows similar profiles of other compounds, especially for the ILMFs of YN and SC that contain similar amounts of sylvestrene, (E)- $\beta$-ocimene, nerol oxide, pinocarvone, $\alpha$-terpinyl acetate and eugenol.

The PCA and clustering results infer that the divergences of the ILMFs in the five regions are due to their different geographic location and environments. YN and SC are located in southwestern China with high altitudes and relatively cold weather. Both FJ and GX are in southern China where the altitude is low, and the weather is relatively warm (Table S1). Therefore, the free and bound volatile profiles of the ILMFs grown in YN and SC are similar and those of FJ and GX are similar. $\mathrm{HB}$ is a province in central China. Its ILMF shows similar free volatiles profile to those of YN and SC and shares bound volatiles profile with those of FJ and GX.

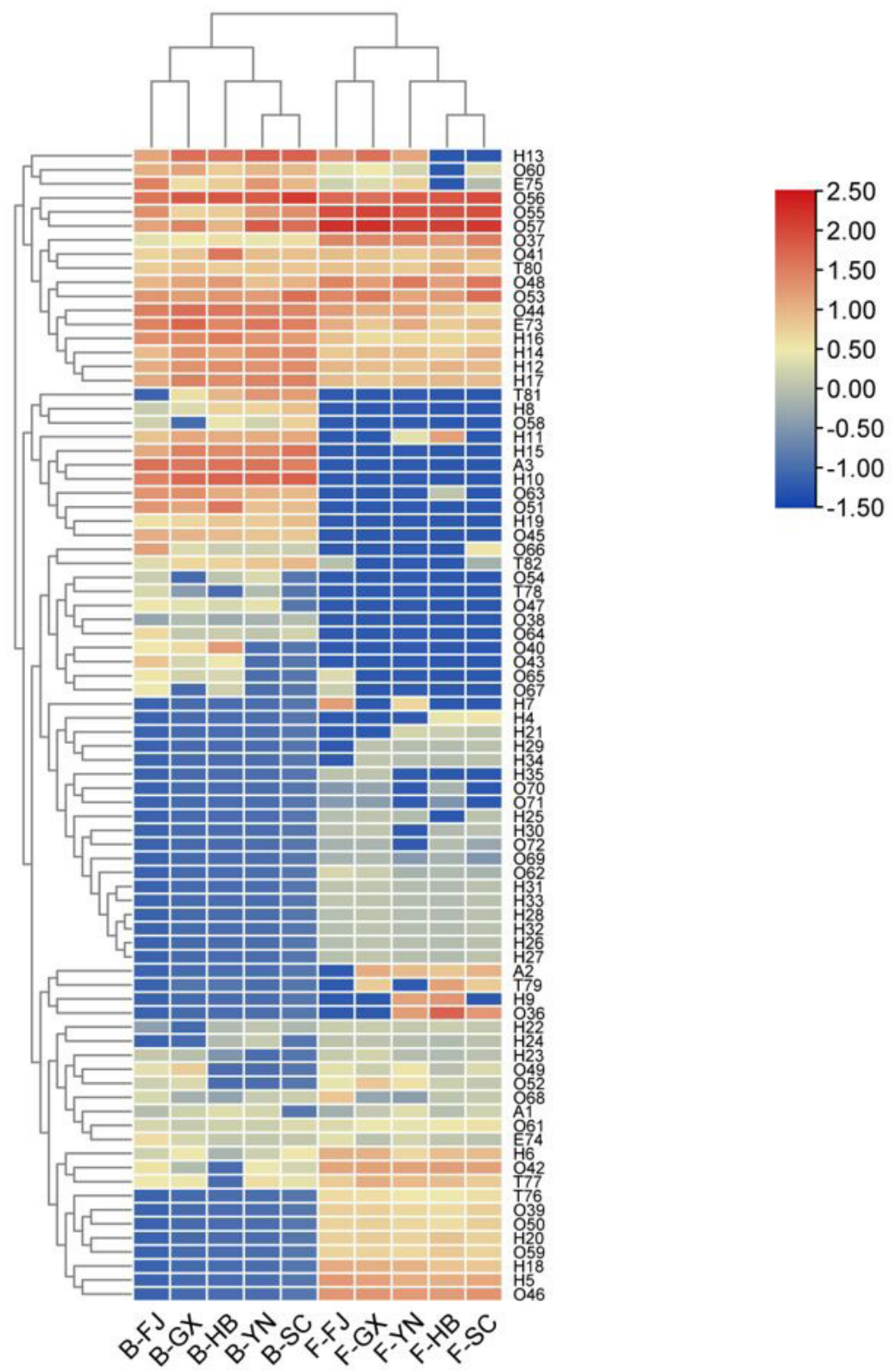

Figure 4. Hierarchical clustering and heatmap visualization of free and bound volatile compounds in ILMFs. The compound numbers are as listed in Table 1 and Table 2. F represensts free volatile compounds and B represents bound volatile compounds. The concentrations of aroma compounds are normalized. 


\section{Conclusions}

In summary, total 82 volatiles with 66 free and 52 bound forms are identified and quantified in the ILMFs harvested in FJ, GX, HB, YN and SC by headspace SPME-GC-MS. Thirty six volatiles are found in both free and bound forms and 67 volatile compounds are detected in Litsea mollis fruit for the first time. Terpene hydrocarbons and oxygenated terpenes are the major volatile compounds in ILMFs. The free aroma compounds are dominated by $\alpha$-pinene, eucalyptol, linalool, $\alpha, \beta$-citral, geraniol and citronellal. $\beta$-Myrcene, $\alpha$-citral, geraniol, citronellal and eugenol are the characteristic bound aroma compounds of ILMFs. The free volatiles of the ILMFs from the five regions share the floral, fruity and herbaceous odor profile. The bound volatiles exhibit similar characteristic odor as the free forms, but with stronger sweet and woody odor, suggesting that the enzymatic hydrolysis can make ILMF sweeter and woodier. The statistical analyses reveal that the volatiles of ILMF is correlated to geographic location. The ILMFs of FJ and GX in southern China show similar free and bound volatiles profiles, and those of YN and SC in southwestern China are similar. The ILMF of HB displays similar free volatiles profile to that of the ILMF in southwestern China, and shares bound volatiles profile with the ILMF of southern China.

\section{Acknowledgements}

This work was funded by the State Key Research and Development Plan "Modern Food Processing and Food Storage and Transportation Technology and Equipment" (No. 2018YFD0400104); the Open Research Fund Program of Beijing Advanced Innovation Center for Food Nutrition and Human Health (No. 20171010) and the Open Research Fund Program of Hubei Key Laboratory of Biologic Resources Protection and Utilization (No. PT012010).

\section{References}

Buettner, A., \& Schieberle, P. (2001). Evaluation of aroma differences between hand-squeezed juices from valencia late and navel oranges by ouantitation of key odorants and flavor reconstitution experiments. Journal of Agricultural and Food Chemistry, 49(5), 2387-2394. http:// dx.doi.org/10.1021/jf0013631. PMid:11368609.

Cai, X., Wang, X., Chen, Y., Wang, Y., Song, D., \& Gu, Q. (2019). A natural biopreservative: antibacterial action and mechanisms of Chinese Litsea mollis Hemsl. extract against Escherichia coli DH5a and Salmonella spp. Journal of Dairy Science, 102(11), 9663-9673. http://dx.doi.org/10.3168/jds.2019-16292. PMid:31447164.

Chen, H. P., Yi, H. X., Liu, Y. Q., \& Zhang, S. Y. (1984). Chemical composition of the essential oil from Litsea mollis Hemsl. fruits. Chinese Traditional and Herbal Drugs, 15(11), 13-15. [in Chinese]

Chinese Academy of Sciences. (2019). Flora of China. Retrieved from http://www.iplant.cn/info/Litsea\%20mollis?t=foc

Chyau, C. C., Ko, P. T., Chang, C. H., \& Mau, J. L. (2003). Free and glycosidically bound aroma compounds in lychee (Litchi chinensis Sonn.). Food Chemistry, 80(3), 387-392. http://dx.doi.org/10.1016/ S0308-8146(02)00278-9.

Davis, E. M., \& Croteau, R. (2000). Cyclization enzymes in the biosynthesis of monoterpenes,sesquiterpenes, and diterpenes. In F. J. Leeper \& J. C. Vederas (Eds.), Biosynthesis: topics in current chemistry (Vol. 209, pp. 53-95). Berlin, Heidelberg: Springer. http:// dx.doi.org/10.1007/3-540-48146-X_2.

Dziadas, M., \& Jelen, H. H. (2016). Comparison of enzymatic and acid hydrolysis of bound flavor compounds in model system and grapes. Food Chemistry, 190, 412-418. http://dx.doi.org/10.1016/j. foodchem.2015.05.089. PMid:26212990.

Elmaci, Y., \& Altug, T. (2005). Flavor characterization of three mandarin cultivars (Satsuma, Bodrum, Clemantine) by using GC/MS and flavor profile analysis techniques. Journal of Food Quality, 28(2), 163-170. http://dx.doi.org/10.1111/j.1745-4557.2005.00009.x.

Fenoll, J., Manso, A., Hell, P., Ruiz, L., \& Flores, P. (2009). Changes in the aromatic composition of the Vitis vinifera grape Muscat Hamburg during ripening. Food Chemistry, 114(2), 420-428. https://doi. org/10.1016/j.foodchem.2008.09.060.

Garcia, C. V., Quek, S. Y., Stevenson, R. J., \& Winz, R. A. (2011). Characterization of the bound volatile extract from baby kiwi (Actinidia arguta). Journal of Agricultural and Food Chemistry, 59(15), 8358-8365. http://dx.doi.org/10.1021/jf201469c. PMid:21702480.

GitHub (2021). CJ-Chen/TBtools. Retrieved from https://github.com/ CJ-Chen/TBtools/releases

Goas, M., Goas, G., \& Larher, F. (1978). Quelques aspects du métabolisme des imino-acides chez les halophytes. Actualités Botaniques, 125(3-4), 259-268. https://doi.org/10.1080/01811789.1978.10826364.

Guimarães, A. C., Meireles, L. M., Lemos, M. F., Guimarães, M. C. C., Endringer, D. C., Fronza, M., \& Scherer, R. (2019). Antibacterial activity of terpenes and terpenoids present in essential oils. Molecules (Basel, Switzerland), 24(13), 2471. http://dx.doi.org/10.3390/ molecules24132471. PMid:31284397.

Huang, D. H., Wang, F. S., Li, Y. H., \& Xiang, J. G. (2014). Chemical composition of the twig oil of Litsea mollis from China. Advanced Materials Research, 997, 136-139. http://dx.doi.org/10.4028/www. scientific.net/AMR.997.136.

Javed, H. U., Wang, D., Shi, Y., Wu, G. F., Xie, H., Pan, Y. Q., \& Duan, C Q. (2018). Changes of free-form volatile compounds in pre-treated raisins with different packaging materials during storage. Food Research International, 107, 649-659. http://dx.doi.org/10.1016/j. foodres.2018.03.019. PMid:29580531.

Kim, K. H., Moon, E., Ha, S. K., Suh, W. S., Kim, H. K., Kim, S. Y., Choi, S. U., \& Lee, K. R. (2014). Bioactive lignan constituents from the twigs of Lindera glauca. Chemical \& Pharmaceutical Bulletin, 62(11), 1136-1140. http://dx.doi.org/10.1248/cpb.c14-00381. PMid:25366316.

Lasekan, O., \& See, N. S. (2015). Key volatile aroma compounds of three black velvet tamarind (Dialium) fruit species. Food Chemistry, 168, 561-565. http://dx.doi.org/10.1016/j.foodchem.2014.07.112. PMid:25172748.

Leffingwell \& Associates. (2020). Odour thresholds tables. Retrieved from http://www.leffingwell.com/odorthre. htm

Liu, Y., He, C., \& Song, H. (2018). Comparison of fresh watermelon juice aroma characteristics of five varieties based on gas chromatographyolfactometry-mass spectrometry. Food Research International, 107, 119-129. http://dx.doi.org/10.1016/j.foodres.2018.02.022. PMid:29580469.

Melgarejo, P., Calm-Sanchez, A., Vazquez-Araujo, L., Hernandez, F., Martinez, J. J., Legua, P., \& Carbonell-Barrachina, A. A. (2011). Volatile composition of pomegranates from 9 Spanish cultivars using headspace solid phase microextraction. Journal of Food Science, 76(1), S114-S120. https://doi.org/10.1111/j.1750-3841.2010.01945.x.

National Institute of Standards and Technology - NIST. (2018). Standard Reference Database (No. 69). Retrieved from http://webbook.nist. gov/chemistry

Niogret, J., Epsky, N. D., Schnell, R. J., Boza, E. J., Kendra, P. E., \& Heath, R. R. (2013). Terpenoid variations within and among half-sibling avocado 
trees, Persea americana Mill. (Lauraceae). PLoS One, 8(9), e73601. http://dx.doi.org/10.1371/journal.pone.0073601. PMid:24039994.

Onawunmi, G. O. (2008). Evaluation of the antimicrobial activity of citral. Letters in Applied Microbiology, 9(3), 105-108. http://dx.doi. org/10.1111/j.1472-765X.1989.tb00301.x.

Pino, J. A., \& Mesa, J. (2006). Contribution of volatile compounds to mango (Mangifera indicaL.) aroma. Flavour and Fragrance Journal, 21(2), 207-213. http://dx.doi.org/10.1002/ffj.1703.

Qian, M. C., \& Wang, Y. (2005). Seasonal variation of volatile composition and odoractivity value of 'Marion' (Rubus spp. hyb) and 'Thornless Evergreen' (R. laciniatus L.)blackberries. Journal of Food Science, 70(1), C13-C20. http://dx.doi.org/10.1111/j.1365-2621.2005.tb09013.x.

Qiu, C., Smuts, J., \& Schug, K. A. (2017). Analysis of terpenes and turpentines using gas chromatography with vacuum ultraviolet detection. Journal of Separation Science, 40(4), 869-877. http:// dx.doi.org/10.1002/jssc.201601019. PMid:27983761.

Sánchez-Palomo, E., Trujillo, M., García Ruiz, A., \& González Viñas, M. A. (2017). Aroma profile of malbec red wines from La Mancha region: chemical and sensory characterization. Food Research International, 100(Pt 1), 201-208. http://dx.doi.org/10.1016/j. foodres.2017.06.036. PMid:28873679.

Sarry, J., \& Gunata, Z. (2004). Plant and microbial glycoside hydrolases: volatile release from glycosidic aroma precursors. Food Chemistry, 87(4), 509-521. http://dx.doi.org/10.1016/j.foodchem.2004.01.003.

Sharma, J., Chatterjee, S., Kumar, V., Variyar, P. S., \& Sharma, A. (2010). Analysis of free and glycosidically bound compounds of ash gourd (Benincasa hispida): Identification of key odorants. Food Chemistry, 122(4), 1327-1332. http://dx.doi.org/10.1016/j.foodchem.2010.03.099.

Shin, E.-C., Craft, B. D., Pegg, R. B., Phillips, R. D., \& Eitenmiller, R. R. (2010). Chemometric approach to fatty acid profiles in Runnertype peanut cultivars by principal component analysis (PCA). Food Chemistry, 119(3), 1262-1270. http://dx.doi.org/10.1016/j. foodchem.2009.07.058.

Stuart, G., Lopes, D., \& Oliveira, J. V. (2001). Deterpenation of Brazilian orange peel oil by vacuum distillation. Journal of the American Oil Chemists' Society, 78(10), 1041-1044. http://dx.doi.org/10.1007/ s11746-001-0385-x.

Swiegers, J. H., \& Pretorius, I. S. (2005). Yeast modulation of wine flavor. Advances in Applied Microbiology, 57, 131-175. http://dx.doi. org/10.1016/S0065-2164(05)57005-9. PMid:16002012.
Van Gemert, L. J. (2011). Odour thresholds. In L. J. Van Gemert (Eds.), Compilations of odour threshold values in air, water and other media (pp. 207-359). Utrecht, The Netherlands: Oliemans Punter \& Partners BV.

Wang, Q., Quan, S., \& Xiao, H. (2019a). Towards efficient terpenoid biosynthesis: manipulating IPP and DMAPP supply. Bioresources and Bioprocessing, 6(1), 1-13. http://dx.doi.org/10.1186/s40643019-0242-z.

Wang, L.-Y., Tian, Y., Qu, Y.-H., Wu, Y.-Z., Li, Y.-C., Li, R., Lin, P.-C., Shang, X.-Y., \& Lin, S. (2019b). Two new terpenoid ester glycosides from the twigs of Litsea cubeba. Journal of Asian Natural Products Research, 20(12), 1129-1136. http://dx.doi.org/10.1080/10286020. 2018.1526789. PMid:30621451.

Whitaker, B., \& Saftner, R. (2000). Temperature-dependent autoxidation of conjugated trienols from apple peel yields 6-methyl-5-hepten-2one, a volatile implicated in induction of scald. Journal of Agricultural and Food Chemistry, 48(6), 2040-2043. http://dx.doi.org/10.1021/ jf991107c. PMid:10888495.

Wu, Y., Duan, S., Zhao, L., Gao, Z., Luo, M., Song, S., Xu, W., Zhang, C., Ma, C., \& Wang, S. (2016). Aroma characterization based on aromatic series analysis in table grapes. Scientific Reports, 6(1), 1-16. http://dx.doi.org/10.1038/srep31116. PMid:27487935.

Wyllie, S. G., \& Fellman, J. K. (2000). Formation of volatile branched chain esters in bananas (Musa sapientum L.). Journal of Agricultural and Food Chemistry, 48(8), 3493-3496. http://dx.doi.org/10.1021/ jf0001841. PMid:10956138.

Xiao, H., Zhang, Y., \& Wang, M. (2019). Discovery and engineering of cytochrome P450s for terpenoid biosynthesis. Trends in Biotechnology, 37(6), 618-631. http://dx.doi.org/10.1016/j.tibtech.2018.11.008. PMid:30528904.

Yang, Y. N., Zheng, F. P., Yu, A. N., \& Sun, B. G. (2019). Changes of the free and bound volatile compounds in Rubus corchorifolius $\mathrm{L}$. f. fruit during ripening. Food Chemistry, 287, 232-240. https://doi. org/10.1016/j.foodchem.2019.02.080.

Yang, Y. N., Liang, M., Yang, Y., Zheng, F. P., Wang, X. P., \& Yu, A. N. (2020). Optimization of a headspace solid-phase microextraction method for the gas chromatography-mass spectrometry analysis aroma compounds of Litsea mollis Hemsl. immature fruit. Food Science and Technology (Campinas), 40(4), 786-793. http://dx.doi. org/10.1590/fst.20319.

Zhang, X. (2015). Spices and food culture in native minorities of Dehong. Agricultural Science and Technology, 16(2), 380-383. [in Chinese] 


\section{Supplementary material}

Supplementary material accompanies this paper.

Legend S1. COMPARISON OF FREE AND BOUND VOLATILE PROFILES OF IMMATURE LITSEA MOLLIS FRUITS GROWN IN FIVE DISTINCT REGIONS OF CHINA.

This material is available as part of the online article from http://www.scielo.br/CTA 